\title{
Combining 3D X-ray Techniques; Computed Tomography and Fluorescence
}

\author{
Brian M. Patterson ${ }^{1}$, Nikolaus Cordes ${ }^{1}$, George J. Havrilla ${ }^{2}$, and Kevin Henderson ${ }^{1}$ \\ 1. Materials Science and Technology Division, Engineered Materials Group, Los Alamos National \\ Laboratory, Los Alamos, NM, USA \\ 2. Chemical Diagnostics and Engineering, Chemistry Division, Los Alamos National Laboratory, Los \\ Alamos, NM, USA
}

X-rays are used in a plethora of techniques to nondestructively study not only the morphology of a material, but also its elemental composition. Nowhere is this indicated better than in the combination of $\mathrm{X}$-ray computed tomography (CT) with X-ray fluorescence (XRF). CT operates at high resolution to obtain morphological information, XRF at lower resolution provides elemental composition information.

X-ray CT illuminates a material with a beam of X-rays that are absorbed by the material, approximately relating to the density, which can be used to collect a radiograph. A series of radiographs are collected as the sample is rotated $180^{\circ}$ which is this reconstructed into a $3 \mathrm{D}$ rendering of the material from which morphological measures can be obtained. The imaging rate depends upon the X-ray flux and can vary from a day or more down to sub-second. No chemical information is obtained, but high resolution (micrometers down to 10's of nanometers) morphological information is collected. CT is used to study a very wide variety of materials including: foams, metals, additive manufactured parts, explosives, animals, etc.

Conversely, X-ray fluorescence illuminates a single spot on the sample in which atoms within the X-ray spot kick off an inner shell electron into which, another electron of the atom takes its place; simultaneously emiting a fluorescent X-ray. The energy of this X-ray is indicative of the element present. The system that we have built has an X-ray optic on both the source and detector which allows for the sequential rastering of the sample through the beam in the $\mathrm{X}, \mathrm{Y}$, and $\mathrm{Z}$ directions, Figure 1. 3D maps of the sample may be collected that can also identify the elements at each location. The resolution of the technique is very coarse compared to CT; it is highly dependent upon the optic (which in turn is also dependent upon the wavelength of the X-ray). The resolution for low energy X-rays ( 2 kev) is 100 micrometers, for higher energy $\mathrm{X}$-rays $(\sim 8 \mathrm{keV})$ is 50 micrometers. This technique can also use the $\mathrm{X}$ rays scattered by low density materials to plot the density variations.

These techniques have been used to understand a variety of material systems [1-4], for this talk we will present the nondestructive analysis of a microSD chip (Figure 2), overlaying the X-ray CT image with the confocal XRF map to better understand the sample's interior morphology and elemental composition.

References:

[1] K. Tsuji, T. Matsuno, Y. Takimoto, M. Yamanashi, N. Kometani, Y. C. Sasaki, et al., Spectrochimica Acta Part B: Atomic Spectroscopy, vol. 113, pp. 43-53, 11/1/ 2015.

[2] N. L. Cordes, S. Seshadri, G. Havrilla, X. Yuan, M. Feser, and B. M. Patterson, Spectrochimica Acta Part B: Atomic Spectroscopy, vol. 103-104, 2015.

[3] N. L. Cordes, G. J. Havrilla, I. O. Usov, K. A. Obrey, and B. M. Patterson, Spectrochimica Acta Part B: Atomic Spectroscopy, vol. 101, pp. 320-329, 2014.

[4] G. J. Havrilla and T. Miller, Powder Diffraction, vol. 19, pp. 119-126, 2012. 

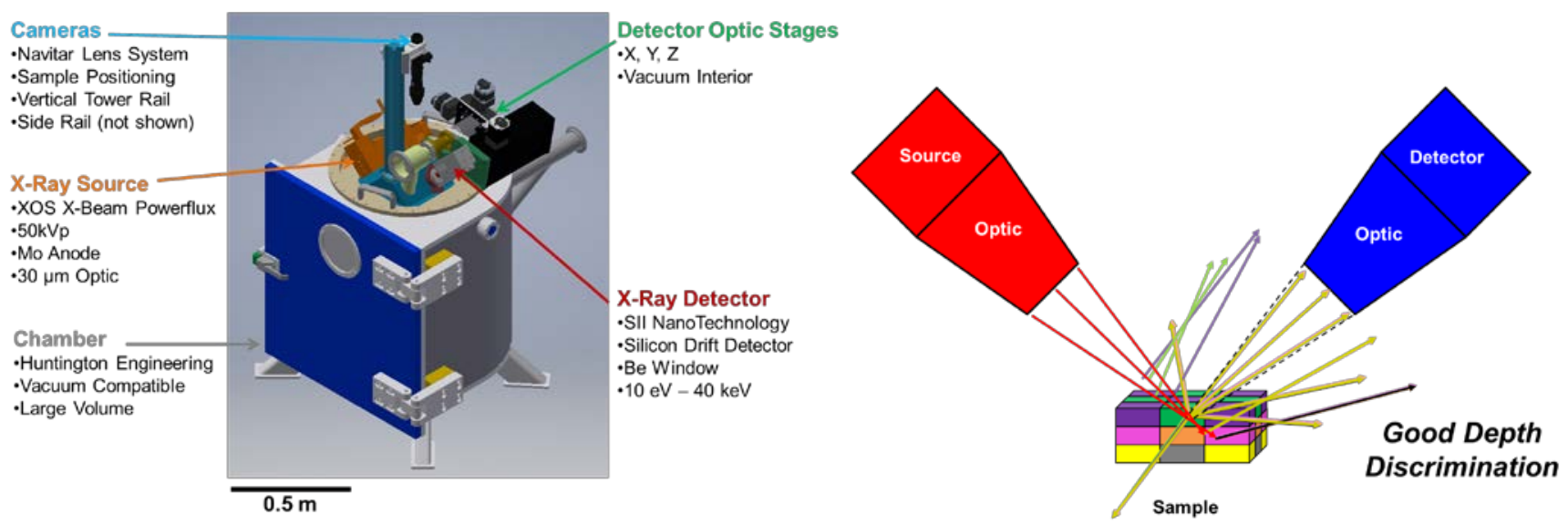

Figure 1. Drawing of our confocal MXRF instrument (left) and cartoon of the geometry (right). The Xray source and detector are mounted on the top, with the optics mounted on the interior. Cameras aid in the sample alignment. An optic on both the source and the detector spatially confine the beam and the detection to an approximately 50 × 50 × 50 micrometer sphere. Each spectrum, at least those farther apart than the spatial resolution, are not spectrally contaminated by overlying and underlying layers.
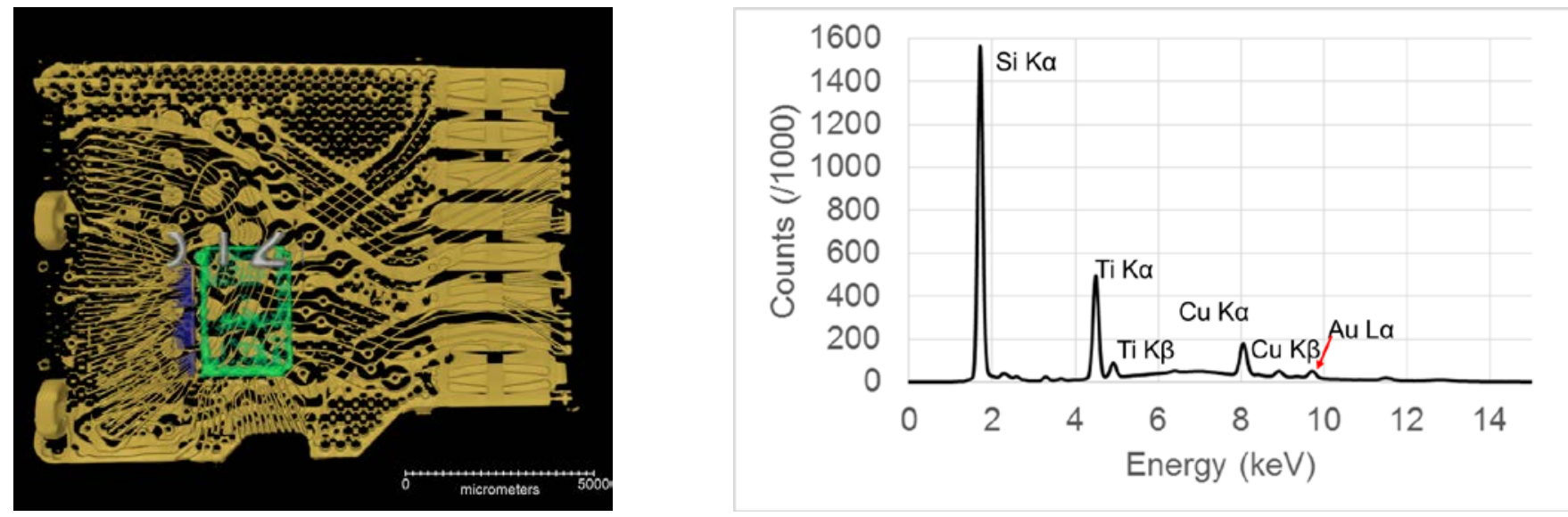

Figure 2. Overlay of X-ray CT image (gold color) with a confocal MXRF image. The MXRF image is colored by the $\mathrm{Cu}$ signal (green), gold (blue) and the Ti dioxide labeling on the chip (white). Only a small region of the chip was analyzed with XRF. The composite XRF spectrum is shown on the right. 